\title{
Ischemic preconditioning maintains the immunoreactivities of glucokinase and glucokinase regulatory protein in neurons of the gerbil hippocampal CA1 region following transient cerebral ischemia
}

\author{
YOUNG SHIN CHO ${ }^{1,2^{*}}$, JUN HWI CHO ${ }^{1 *}$, BICH-NA SHIN $^{3}$, GEUM-SIL CHO $^{4}$, IN HYE KIM $^{5}$, \\ JOON HA PARK ${ }^{5}$, JI HYEON AHN ${ }^{5}$, TAEK GEUN OHK ${ }^{1,6}$, BYUNG-RYUL CHO ${ }^{7}$, \\ YOUNG-MYEONG KIM ${ }^{8}$, SEONGKWEON HONG ${ }^{9}$, MOO-HO WON ${ }^{5}$ and JAE-CHUL LEE ${ }^{5}$
}

\footnotetext{
${ }^{1}$ Department of Emergency Medicine, School of Medicine, Kangwon National University, Chuncheon, Gangwon 200-701;

${ }^{2}$ Department of Emergency Medicine, Seoul Hospital, College of Medicine, Sooncheonhyang University, Seoul 140-743;

${ }^{3}$ Department of Physiology, College of Medicine and Institute of Neurodegeneration and Neuroregeneration, Hallym University, Chuncheon, Gangwon 200-702; ${ }^{4}$ Department of Neuroscience, College of Medicine, Korea University, Seoul 136-705;

${ }^{5}$ Department of Neurobiology, School of Medicine, Kangwon National University, Chuncheon, Gangwon 200-701;

${ }^{6}$ Department of Emergency Medicine, Kangnam Sacred Heart Hospital, College of Medicine, Hallym University, Seoul 150-950; Departments of ${ }^{7}$ Internal Medicine and ${ }^{8}$ Molecular and Cellular Biochemistry and ${ }^{9}$ Surgery, School of Medicine, Kangwon National University, Chuncheon, Gangwon 200-701, Republic of Korea
}

Received September 24, 2014; Accepted June 15, 2015

DOI: $10.3892 / \mathrm{mmr} .2015 .4021$

\begin{abstract}
Glucokinase (GK) is involved in the control of blood glucose homeostasis. In the present study, the effect of ischemic preconditioning (IPC) on the immunoreactivities of $\mathrm{GK}$ and its regulatory protein (GKRP) following 5 min of transient cerebral ischemia was investigated in gerbils. The gerbils were randomly assigned to four groups (sham-operated group, ischemia-operated group, IPC + sham-operated group and IPC + ischemia-operated group). IPC was induced by subjecting the gerbils to $2 \mathrm{~min}$ of ischemia, followed by 1 day of recovery. In the ischemia-operated group, a significant loss of neurons was observed in the stratum pyramidale (SP) of the hippocampal CA1 region (CA1) at 5 days post-ischemia; however, in the IPC+ischemia-operated group, the neurons in the SP were well protected. Following immunohistochemical investigation, the immunoreactivities of GK and GKRP in the neurons of the
\end{abstract}

Correspondence to: Professor Moo-Ho Won or Dr Jae-Chul Lee, Department of Neurobiology, School of Medicine, Kangwon National University, 1 Kangwondaehak Street, Chuncheon, Gangwon 200-701, Republic of Korea

E-mail: mhwon@kangwon.ac.kr

E-mail: anajclee@kangwon.ac.kr

${ }^{*}$ Contributed equally

Key words: ischemic preconditioning, ischemia-reperfusion, delayed neuronal death, glucokinase, glucokinase regulatory protein
SP were markedly decreased in the CA1, but not the CA2/3, from 2 days post-ischemia, and were almost undetectable in the SP 5 days post-ischemia. In the IPC + ischemia-operated group, the immunoreactivities of GK and GKRP in the SP of the CA1 were similar to those in the sham-group. In brief, the findings of the present study demonstrated that IPC notably maintained the immunoreactivities of GK and GKRP in the neurons of the SP of CA1 following ischemia-reperfusion. This indicated that GK and GKRP may be necessary for neuron survival against transient cerebral ischemia.

\section{Introduction}

Brain ischemia results in the deprivation of oxygen and glucose in brain tissue, which may lead to permanent brain damage. Several clinical conditions can give rise to global cerebral ischemia; a frequent cause is cardiac arrest, which is a significant worldwide health problem (1-3). Mongolian gerbils are widely used as an animal model of chronic or transient cerebral ischemia, as $\sim 90 \%$ of the gerbils lack the communicating vessels between the carotid and vertebral arteries (4). An important feature of cerebral ischemic damage is the vulnerability of specific neuronal populations. In particular, pyramidal neurons in the hippocampal CA1 region do not die immediately but survive for several days, which is referred to as 'delayed neuronal death' (5). It is well known that the delayed neuronal death is associated with multiple mechanisms, including changes in energy metabolism, oxidative stress, neurotoxicity and neuroinflammation (6-9). However, the exact mechanisms underlying neuronal damage in ischemia and delayed neuronal death remain to be fully elucidated. 
Ischemic preconditioning (IPC) represents an important adaptation of the central nervous system to sub-lethal ischemia, which results in an increased tolerance to ischemia in the central nervous system to a subsequent longer or lethal period of ischemia $(10,11)$. IPC induces the expression of a diverse family of genes involved in cytoprotection, which, in turn, encode proteins that enhance brain resistance to ischemia (12). This mechanism has been termed 'ischemic tolerance,' and the basic mechanisms underlying ischemic tolerance remain to be fully elucidated (13).

Glucokinase (GK), a member of the hexokinase family, catalyzes the ATP-dependent phosphorylation of glucose to produce glucose-6-phosphate. GK is expressed predominantly in the liver, pancreatic $\beta$-cells and brain (14-17), and its activity is generally modulated by glucokinase regulatory protein (GKRP), which is stimulated by binding fructose-6-phosphate and suppressed by binding fructose-1-phosphate (18-20). GK and GKRP are important in the regulation of glucose homeostasis, as glucose sensors involved in the control of food intake (18,21). Certain studies have suggested an association between neuronal cell death and glucose dysregulation $(22,23)$. In addition, our previous study reported that changes in the expression of GK and GKRP, which are expressed in the hippocampus, were associated with neuronal loss (24). However, the expression patterns of GK and GKRP in the brain of IPC-mediated animals following transient cerebral ischemia remain to be fully elucidated.

Therefore, the present study was performed to examine whether GK and GKRP are associated with IPC-induced neuroprotection, which has been widely accepted by a number of previous studies $(11,12)$, following transient cerebral ischemia in the hippocampus of the gerbil, which is considered a suitable animal model of transient cerebral ischemia $(25,26)$.

\section{Materials and methods}

Experimental animals. The present study used male Mongolian gerbils (Meriones unguiculatus), obtained from the Experimental Animal Center, Kangwon National University (Chuncheon, South Korea). The gerbils used were 6 months of age (body weight, 65-75 g). The animals were housed in a conventional state under suitable temperature $\left(23^{\circ} \mathrm{C}\right)$ and humidity $(60 \%)$ control, with a 12-h light/12-h dark cycle, and were provided with free access to food and water. All experimental protocols were approved by the Institutional Animal Care and Use Committee at Kangwon University (approval no. KW-130424-1) and adhered to guidelines that are in compliance with the current international laws and policies (Guide for the Care and Use of Laboratory Animals). All the experiments were performed in a manner to minimize the number of animals used and the suffering caused by the procedures used in the present study.

Induction of transient cerebral ischemia in animal groups. The animals were divided into four groups: i) sham-operated group (sham group; $n=7$ at each time point), in which the bilateral common carotid arteries were exposed, but no ischemia was induced (sham-surgery) in the animals; ii) ischemia-operated group (ischemia group; $n=7$ at each time-point), in which the animals were exposed to $5 \mathrm{~min}$ of transient ischemia; iii) IPC + sham-operated group (IPC + sham group; $n=7$ at each time- point), in which the animals were subjected to a 2 min sublethal ischemic insult prior to sham surgery; and iv) IPC + ischemia-operated group (IPC + ischemia group; $\mathrm{n}=7$ at each time-point), in which the animals were subjected to a 2 min sublethal ischemic insult prior to $5 \mathrm{~min}$ of transient ischemia. The preconditioning paradigm has been previously confirmed to be effective at protecting neurons against ischemia in this ischemic model (27). The animals in the ischemia group and the IPC + ischemia groups were assigned recovery durations of 1, 2 and 5 days, as pyramidal neurons in the hippocampal CA1 region do not die until 3 days and begin to die 4 days after ischemia-reperfusion (5).

Transient cerebral ischemia was developed according to our previously described method (9). The experimental animals were anesthetized using a mixture of $2.5 \%$ isoflurane (Baxter, Deerfield, IL, USA) in 33\% oxygen and $67 \%$ nitrous oxide (Chil-Seung Gas Co., Gyeonggi-do, Korea). Under an operating microscope (Boom Stand Zoom trinocular microscope, Shanghai Optical Instrument Factory, Shanghai, China), a ventral neck incision was made and the bilateral common carotid arteries were gently exposed. Ischemia was induced by occluding the arteries using non-traumatic aneurysm clips (Yasargil FE 723 K; Aesculap, Tuttlingen, Germany). The complete interruption of blood flow was confirmed by observing the central artery in the retinae using an ophthalmoscope (HEINE K180 ${ }^{\circledR}$; Heine Optotechnik, Herrsching, Germany). Following occlusion for 2 or $5 \mathrm{~min}$, the aneurysm clips were removed from the common carotid arteries. The body (rectal) temperature under free-regulating or normothermic $\left(37 \pm 0.5^{\circ} \mathrm{C}\right)$ conditions was monitored using a rectal temperature probe (TR-100; Fine Science Tools, Foster City, CA) and maintained using a thermometric blanket prior to, during and following surgery, until the animals had completely recovered from anesthesia. Subsequently, the animals were maintained on the thermal incubator (temperature, $23^{\circ} \mathrm{C}$; humidity, 60\%; Mirae Medical Industry, Seoul, South Korea) to maintain the body temperature of animals until the animals were sacrificed.

Tissue processing for histology. All the animals were anesthetized with pentobarbital sodium and perfused transcardially with $0.1 \mathrm{M}$ phosphate-buffered saline (PBS; $\mathrm{pH}$ 7.4) followed by $4 \%$ paraformaldehyde (Samchun Pure Chemical Co., Ltd., Gyeonggi-do, Korea) in 0.1 M phosphate-buffer (PB; pH 7.4). The brains were removed and post-fixed in the same fixative for $6 \mathrm{~h}$. The brain tissues were cryoprotected by infiltration with $30 \%$ sucrose overnight. The frozen tissues were then serially sectioned on a cryostat (CM1900 UV; Leica, Wetzlar, Germany) into $30 \mu \mathrm{m}$ coronal sections, and were collected into six-well plates containing PBS.

Cresyl violet $(C V)$ staining. The levels of neuronal death in the hippocampal CA1 region in each group were examined using CV staining. The sections were mounted on gelatin-coated microscope slides. Cresyl violet acetate (Sigma-Aldrich, St. Louis, MO) was dissolved at $1.0 \%$ (w/v) in distilled water, and glacial acetic acid (Marienfeld-Superior, Lauda-Königshofen, Germany) was added to this solution. The sections were stained and dehydrated by immersion in serial ethanol baths, and were 
then mounted using Canada balsam (Kanto Chemical, Co., Inc., Tokyo, Japan).

Neuronal nuclei (NeuN) immunohistochemistry. To examine the neuronal changes in the hippocampal CA1 region following transient cerebral ischemia using anti-NeuN, a marker for neurons, the sections were sequentially treated with $0.3 \%$ hydrogen peroxide $\left(\mathrm{H}_{2} \mathrm{O}_{2}\right)$ in PBS for $30 \mathrm{~min}$ and $10 \%$ normal goat serum in $0.05 \mathrm{M}$ PBS for $30 \mathrm{~min}$. The sections were then incubated with diluted mouse anti-NeuN, a neuron-specific soluble nuclear antigen, (1:1,000; Chemicon International, Temecula, CA) overnight at at $4^{\circ} \mathrm{C}$. Subsequently, the tissues were exposed to biotinylated horse anti-mouse IgG and streptavidin peroxidase complex (Vector Laboratories, Inc., Burlingame, CA). The sections were then visualized by staining with $0.05 \% 3,3$ '-diaminobenzidine in $0.1 \mathrm{M}$ Tris- $\mathrm{HCl}$ buffer and mounted on the gelatin-coated slides. Following dehydration, the sections were mounted using Canada balsam (Kanto Chemical, Co., Inc.).

Fluoro-Jade B (F-J B) histofluorescence. To examine neuronal death in the CA1 region at each time-point following ischemia, F-J B, a high affinity fluorescent marker for the localization of neuronal degeneration, histofluorescence was performed (28). The sections were first immersed in a solution containing $1 \%$ sodium hydroxide in $80 \%$ alcohol, followed by immersion in $70 \%$ alcohol. The sections were then transferred into a solution of $0.06 \%$ potassium permanganate, followed by transfer to a $0.0004 \%$ F-J B (Histochem, Jefferson, AR, USA) staining solution. Following washing with PBS three times, the sections were placed on a slide warmer at $\sim 50^{\circ} \mathrm{C}$, and then examined using an epifluorescent microscope (LSM510 META NLO; Carl Zeiss, Göttingen, Germany) with blue (450-490 nm) excitation light and a barrier filter. Using this method, neurons that undergo degeneration fluoresce brightly, compared with the background (10).

Cell counts. All measurements were performed in a blinded-manner, to ensure objectivity, by three observers for each experiment, with measurements of experimental samples under the same conditions. According to anatomical landmarks corresponding to AP, between -1.4 and $-1.9 \mathrm{~mm}$ of the gerbil brain atlas, the tissue sections were selected with a $300-\mu \mathrm{m}$ interval, and cell counts were obtained by averaging the total numbers of cells in 15 sections from each animal in each group. The numbers of NeuN- and F-J B-positive cells were counted in a 200x200 $\mu \mathrm{m}$ square, which was centred approximately at the center of the CA1 region using an image analyzing system (Optimas 6.5; CyberMetrics, Scottsdale, AZ, USA). The cell counts were obtained by averaging the total number from each animal per group.

Immunohistochemistry for GK and GKRP. To obtain accurate data for immunoreactivity, the sections from the sham-operated, IPC + sham, ischemia-operated and IPC + ischemia groups ( $\mathrm{n}=7$ at each time-point) were stained, according to the above-mentioned NeuN immunohistochemical staining. The sections were incubated with diluted rabbit anti-GK (1:200; Santa Cruz Biotechnology, Inc., Santa Cruz, CA, USA) and goat anti-GKRP (1:200; Santa Cruz Biotechnology, Inc.), followed by incubation with and subsequently biotinylated horse anti-rabbit or anti-goat IgG and streptavidin peroxidase complex (1:200; Vector Laboratories, Inc.). In order to establish the specificity of the immunostaining, a negative control was included, in which pre-immune serum was used in place of the primary antibody. The negative control resulted in the absence of immunoreactivity in any structures.

A total of 20 sections per animal were selected to quantitatively analyze the immunoreactivities of GK and GKRP. The cellular immunoreactivities of GK and GKRP were graded in the hippocampal CA1 region. Digital images of the CA1 region, including the strata oriens, pyramidale and radiatum in the hippocampus proper, were captured using an AxioM1 light microscope (Carl Zeiss) equipped with a digital camera (MRc5; Axiocam, Carl Zeiss) connected to a PC monitor. Semi-quantification of the intensity of GK+ and GKRP+ structures were evaluated using digital image analysis software (MetaMorph 4.01; Universal Imaging Corp.). The level of immunoreactivity was scaled as,,- \pm+ or ++ , representing no staining (gray scale value, $\geq 200$ ), weakly positive staining (gray scale value, 150-199), moderate staining (gray scale value, 100-149), or marked staining (gray scale value, s99) (29).

Statistical analysis. All data are presented as the mean \pm standard error of the mean. A multiple-sample comparison was performed to compared the differences between groups, using analysis of variance. Tukey's multiple range test was used as a post-hoc test, using the criterion of the least significant differences. SAS version 9.2 (SAS Institute Inc., Cary, NC, USA) was used to perform the analyses. $\mathrm{P}<0.05$ was considered to indicate a statistically significant difference.

\section{Results}

Determination of neuronal damage using cresyl violet-positive $\left(\mathrm{CV}^{+}\right)$cells. The present study examined whether IPC was associated with an increase in neuronal survival in the hippocampal CA1 region following ischemia. In the sham group, the $\mathrm{CV}^{+}$cells were readily observed in all subregions of the hippocampus (Fig. 1A and B). Neurons in the stratum pyramidale (SP) were relatively large and pyramid-like or round in shape. In the IPC + sham group, the CA1 pyramidal cells were well stained with CV (Fig. 1C and D). At 5 days post-ischemia-reperfusion, the number of $\mathrm{CV}^{+}$cells in the sham group was significantly decreased in the SP of the CA1 region, but not the $\mathrm{CA} 2 / 3$ region, compared with those of the ischemia group (Fig. 1E). The damaged cells were shrunken and contained dark and polygonal nuclei (Fig. 1F).

In the IPC + ischemia group, the distribution pattern of $\mathrm{CV}^{+}$ cells in the SP was similar to that observed in the IPC + sham group 5 days after ischemia-reperfusion (Fig. $1 \mathrm{G}$ and $\mathrm{H}$ ).

$\mathrm{NeuN}^{+}$and $\mathrm{F}-\mathrm{J} \mathrm{B}^{+}$neurons. The protection afforded by IPC in the CA1 region was assessed using NeuN immunohistochemistry and F-J B histofluorescence staining (Fig. 2). In the sham group, the CA1 pyramidal neurons were well-stained with NeuN (Table I; Fig. 2A); however, no F-J B ${ }^{+}$neurons were identified (Table I; Fig. 2B). In the IPC + sham group, pyramidal neurons in the $\mathrm{CA} 1$ region were also well-stained with 
Table I. Changes in the mean number of pyramidal neurons of the hippocampal CA1 region of gerbils in the ischemia and IPC + ischemia groups.

\begin{tabular}{|c|c|c|c|c|}
\hline \multirow{2}{*}{$\begin{array}{l}\text { Time post-I-R } \\
\text { (days) }\end{array}$} & \multicolumn{2}{|c|}{ Ischemia-operated group } & \multicolumn{2}{|c|}{ IPC + ischemia-operated group } \\
\hline & $\mathrm{NeuN}^{+}$ & F-J B ${ }^{+}$ & $\mathrm{NeuN}^{+}$ & F-J B ${ }^{+}$ \\
\hline Sham (no I-R) & $346 \pm 13.54$ & 0 & $352 \pm 16.28$ & 0 \\
\hline 1 & $352 \pm 11.12$ & 0 & $349 \pm 18.36$ & 0 \\
\hline 2 & $349 \pm 12.98$ & $4 \pm 4.36^{\mathrm{a}}$ & $355 \pm 17.34$ & 0 \\
\hline 5 & $41 \pm 8.35^{\mathrm{a}, \mathrm{b}}$ & $134 \pm 17.69^{\mathrm{a}, \mathrm{b}}$ & $324 \pm 15.85^{\mathrm{a}, \mathrm{b}}$ & $24 \pm 5.54^{\mathrm{a}, \mathrm{b}}$ \\
\hline
\end{tabular}

Numbers of NeuN ${ }^{+}$and F-J B ${ }^{+}$cells were counted in a 200x200 $\mu \mathrm{m}$ square of the stratum pyramidale of the CA1 region following I-R (n=7 per group; ${ }^{\mathrm{P}}<0.05$, compared with the sham group; ${ }^{\mathrm{b}} \mathrm{P}<0.05$, compared with the 2 days). I-R, ischemia-reperfusion; IPC, ischemic preconditioning .
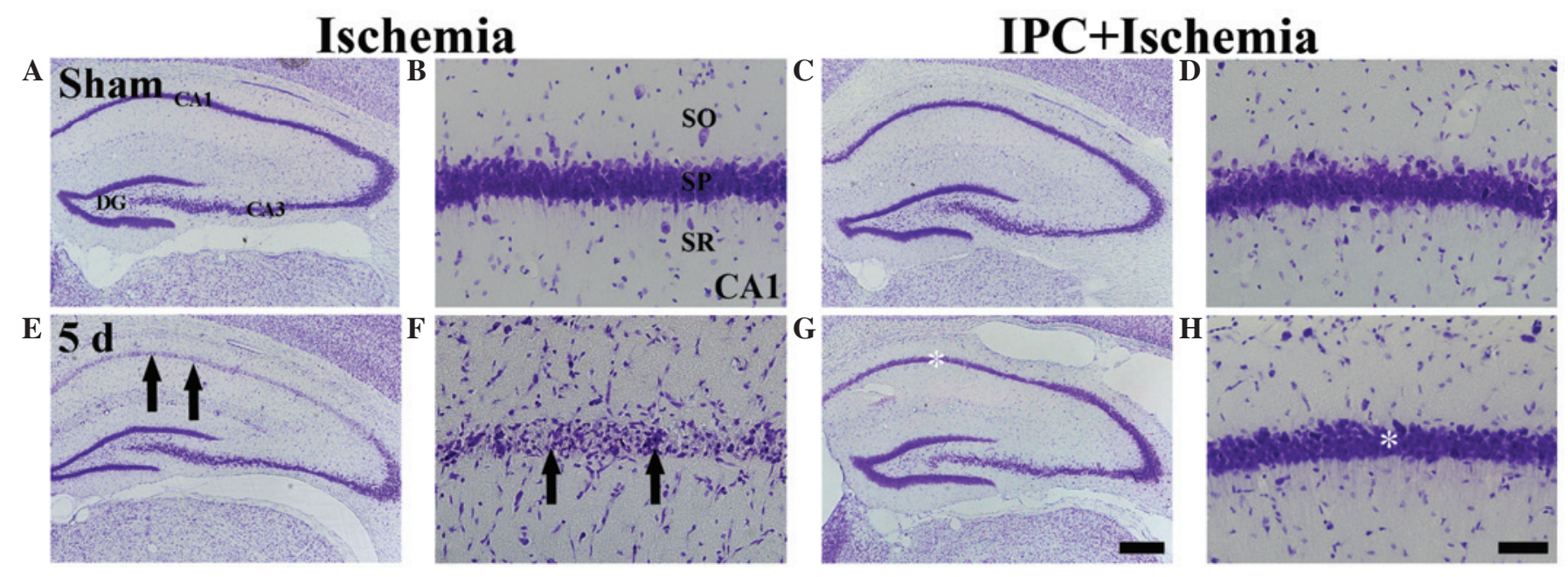

Figure 1. CV histochemical staining in the CA1 region of the ischemia (left two columns) and IPC + ischemia (right two columns) groups at (A-D) sham and $(\mathrm{E}-\mathrm{H}) 5$ days post-ischemia-reperfusion. In the ischemia group, few $\mathrm{CV}^{+}$cells (arrows) were observed in the $\mathrm{SP} 5$ days post-ischemia, whereas $\mathrm{CV}^{+}$cells were abundant (white asterisk) in the SP of the IPC + ischemia group. Scale bar=800 $\mu \mathrm{m}$. (A, C, E, G) and $50 \mu \mathrm{m}$. (B, D, F, H). CV, Cresyl violet; SP, stratum pyramidale; DG, dentate gyrus; SO, stratum oriens; SR, stratum radiatum.

NeuN (Fig. 2C), and no F-J B cells were observed (Table I; Fig. 2C and D).

At 5 days post-ischemia, the number of $\mathrm{NeuN}^{+}$neurons were markedly decreased in the SP of the CA1 region (Table I; Fig. 2E) and several F-J B ${ }^{+}$cells were detected in the SP of the CA1 region (Table I, Fig. 2F).

In the IPC + ischemia group, no significant difference was observed in the distribution patterns of the $\mathrm{NeuN}^{+}$and F-J $\mathrm{B}^{+}$neurons in the SP 5 day after ischemia-reperfusion, compared with those in the IPC + sham group (Table I; Fig. 2G and $\mathrm{H})$.

GK immunoreactivity. In the sham group, marked GK immunoreactivity was detected in neurons of the SP of the CA1-3 regions (Table II; Fig. 3A). In the ischemia group, GK immunoreactivity was altered in the CA1 region, but not in the CA2/3 region. At 1 day-post ischemia-reperfusion, GK immunoreactivity in the SP was not significantly different, compared with that in the sham group (Table II; Fig. 3B). However, GK immunoreactivity in the SP was distinctively decreased 2 days after ischemia-reperfusion (Table II; Fig. 3C). At 5 days-post ischemia-reperfusion, GK immunoreactivity was almost undetected in the pyramidal neurons of the SP (Table II; Fig. 3D). However, in the CA2/3 region, no significant changes in GK immunoreactivity were observed following ischemia-reperfusion (Table II).

In the IPC + sham group, marked GK immunoreactivity was readily detected in the SP of the CA1-3 regions (Table II; Fig. 3E). In the IPC + ischemia-group, GK immunoreactivity in the SP of the CA1-3 regions was well-maintained following ischemia-reperfusion (Table II; Fig. 3F-H).

GKRP immunoreactivity. In the sham group, marked GKRP immunoreactivity was detected, predominantly in the SP of the CA1-3 regions. In this group, moderate GKRP immunoreactivity was observed in cells of the strata oriens and radiatum (Table II; Fig. 4A). In the ischemia groups, GKRP immunoreactivity was altered in the CA1 region, but notin the $\mathrm{CA} 2 / 3$ region. At 1 day post-ischemia-reperfusion, no change in GKRP immunoreactivity was observed in the SP of the CA1 region, compared with that in the sham group (Table II; Fig. 4B). However, GKRP immunoreactivity in the SP was marginally decreased 2 days after ischemia-reperfusion (Table II; Fig. 4C). At 5 days post-ischemia-reperfusion, 
Table II. Semi-quantifications of the immunoreactivities of GK and GKRP in the hippocampal CA1 and CA2/3 regions in the ischemia and IPC + ischemia groups.

Time following ischemia-reperfusion

\begin{tabular}{|c|c|c|c|c|c|c|c|}
\hline Antibody & Region & Group & Category & Sham & 1 day & 2 days & 5 days \\
\hline \multirow[t]{8}{*}{ GK } & CA1 & Ischemia & CSP & ++ & ++ & + & \pm \\
\hline & & & CSOR & + & \pm & \pm & \pm \\
\hline & & IPC+ischemia & CSP & ++ & ++ & ++ & ++ \\
\hline & & & CSOR & \pm & \pm & \pm & \pm \\
\hline & $\mathrm{CA} 2 / 3$ & Ischemia & CSP & ++ & ++ & ++ & ++ \\
\hline & & & CSOR & \pm & \pm & \pm & \pm \\
\hline & & IPC+ischemia & CSP & ++ & ++ & ++ & ++ \\
\hline & & & CSOR & \pm & \pm & \pm & \pm \\
\hline \multirow[t]{8}{*}{ GKRP } & CA1 & Ischemia & CSP & ++ & ++ & + & \pm \\
\hline & & & CSOR & + & + & + & ++ \\
\hline & & IPC+ischemia & CSP & ++ & ++ & ++ & ++ \\
\hline & & & CSOR & + & + & + & + \\
\hline & $\mathrm{CA} 2 / 3$ & Ischemia & CSP & ++ & ++ & ++ & ++ \\
\hline & & & CSOR & + & + & + & + \\
\hline & & IPC+ischemia & CSP & ++ & ++ & ++ & ++ \\
\hline & & & CSOR & + & + & + & + \\
\hline
\end{tabular}

Immunoreactivity is scaled as -,, \pm+ or ++ , representing no staining, weakly positive staining, moderate staining, or marked staining, respectively. Sham, no ischemia reperfusion; CSP, cells in stratum pyramidale; CSOR, cells in stratum oriens and radiatum; IPC, ischemic preconditioning.

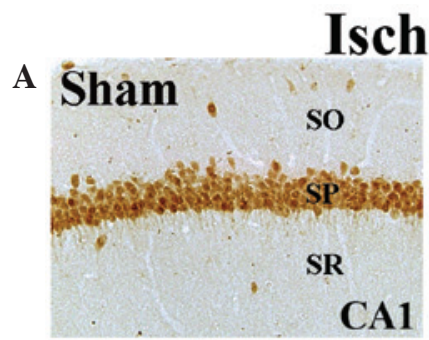

$\mathbf{E}$

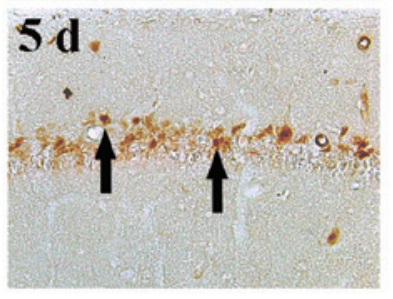

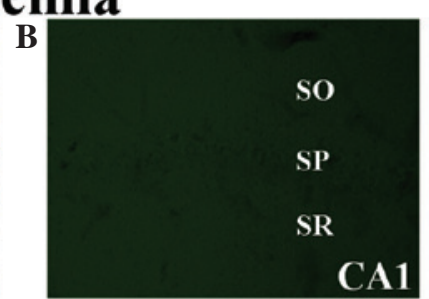

F

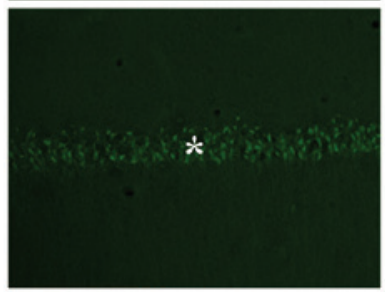

IPC+Ischemia

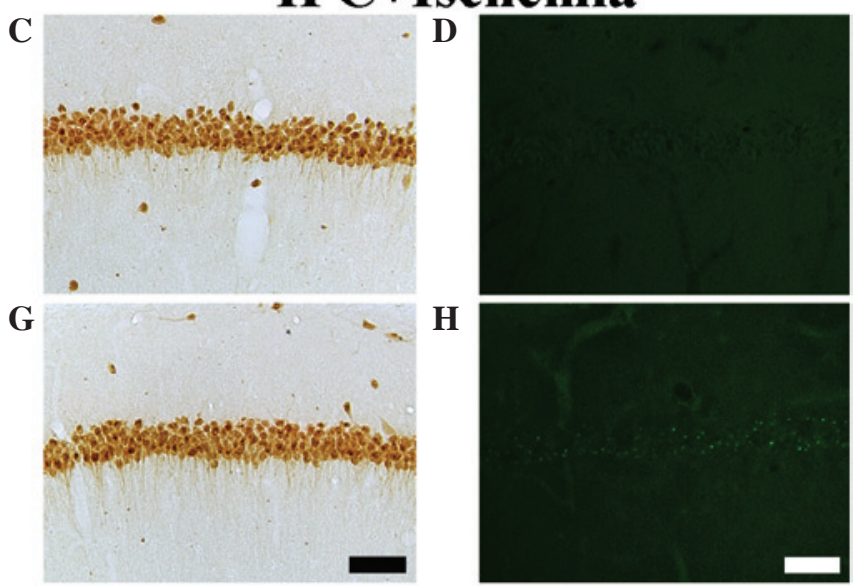

Figure 2. NeuN immunohistochemistry (the first and third columns) and F-J B histofluorescence staining (the second and fourth columns) in the CA1 region of the ischemia group (left two columns) and IPC + ischemia group (right two columns) at (A-D) sham and (E-H) 5 days-post ischemia-reperfusion. In the sham group, several $\mathrm{NeuN}^{+}$neurons and no F-J B $\mathrm{B}^{+}$cells were detected in the SP. In the ischemia group, a few NeuN ${ }^{+}$neurons (black arrows) and several F-J $\mathrm{B}^{+}$ cells (white asterisks) were detected in the $\mathrm{SP}$ at 5 days post-ischemia. However, in the IPC + ischemia-group, abundant NeuN ${ }^{+}$neurons and no F-J $\mathrm{B}^{+}$cells were detected in the SP at 5 days post-ischemia. Scale bar=50 $\mu \mathrm{m}$. NeuN, neuronal nuclei; F-J B, fluoro-Jade B; IPC, ischemic preconditioning; SP, stratum pyramidale; SO; stratum oriens, SR; stratum radiatum.

GKRP immunoreactivity was notably decreased in the SP of the CA1 region; however, at this time-point, marked GKRP immunoreactivity was observed in several non-pyramidal cells in the strata oriens and radiatum (Table II; Fig. 4D). In the CA2/3 region, no significant changes were identified in GKRP immunoreactivity following ischemia-reperfusion (Table II).
In the IPC + sham group, marked GKRP immunoreactivity in the CA1-3 regions was well-detected in the neurons of the SP, and moderate GKRP immunoreactivity was observed in the cells of the strata oriens and radiatum (Table II; Fig. 4E). In the IPC + ischemia group, no significant changes in GKRP immunoreactivity were observed in the CA1-3 regions following ischemia-reperfusion (Table II; Fig. 4F-H). 


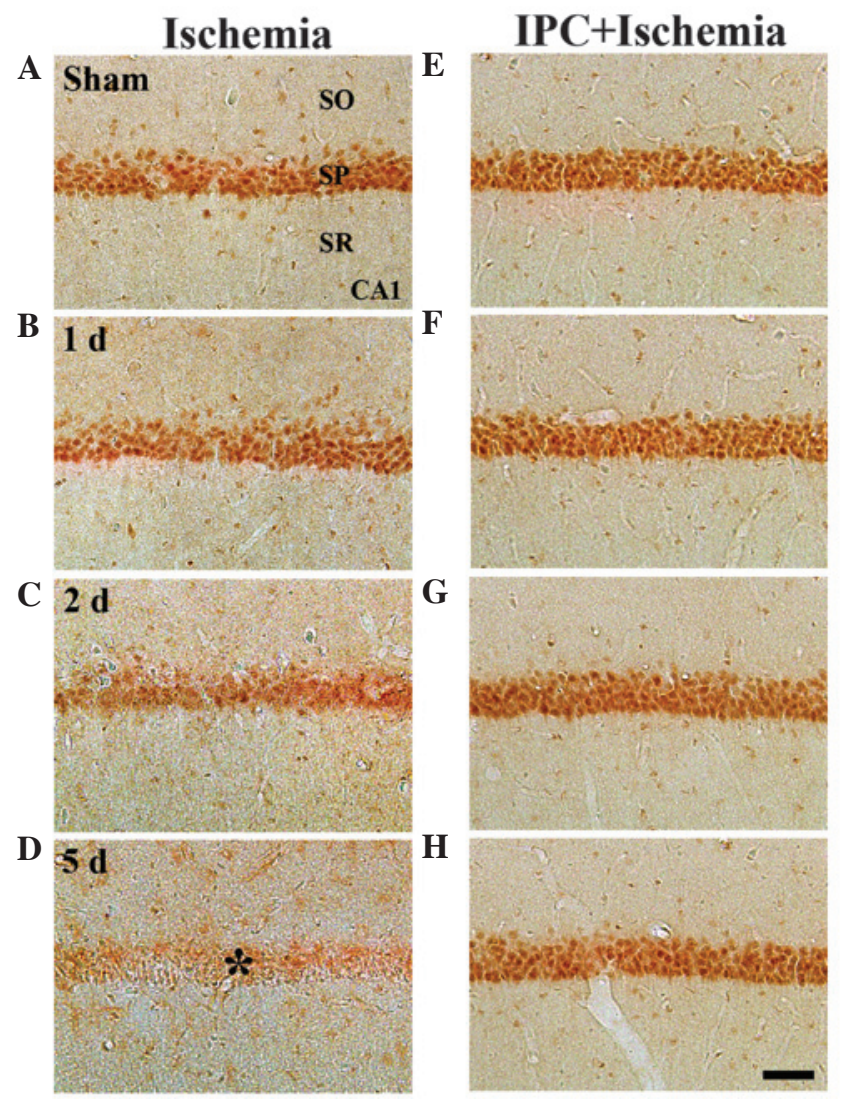

Figure 3. Immunohistochemistry to determine the expression of GK in the CA1 region of the (A-D) ischemia and (E-H) IPC + ischemia groups 1,2 and 5 days following ischemia-reperfusion. GK immunoreactivity was readily detected in the SP in the sham group. GK immunoreactivity in the SP was decreased from 2 days post-ischemia, and GK immunoreactivity was markedly decreased $(*) 5$ days post-ischemia. However, in the IPC-operated sham- and IPC + ischemia-operated groups, GK immunoreactivity was markedly increased in the SP of CA1, and was maintained until 5 days post-ischemia. Scale bar $=50 \mu \mathrm{m}$. GK, glucokinase; SP, stratum pyramidale; SO; stratum oriens, SR; stratum radiatum; IPC, ischemic preconditioning.

\section{Discussion}

GK is important in the control of blood glucose homeostasis. In the present study, the effect of IPC on the immunoreactivities of GK and its regulatory protein GKRP were examined, following $5 \mathrm{~min}$ of transient cerebral ischemia in gerbils. The gerbils were randomly assigned into a sham-operated group, ischemia-operated group, IPC + sham-operated group and IPC + ischemia-operated group. IPC was induced by subjecting the gerbils to 2 min of ischemia followed by 1 day of recovery. In the immunohistochemical investigation, the immunoreactivities of the GK and GKRP in neurons of the SP were distinctively decreased in the CA1, but not the CA2/3, from 2 days post-ischemia, and weer almost undetectable in the SP 5 days post-ischemia. In the IPC + ischemia-operated group, the immunoreactivities of GK and GKRP in the SP of the CA1 were similar to those in the sham group. Therefore, these findings demonstrated that IPC maintains immunoreactivities of GK and GKRP in neurons of the SP of the CA1 following ischemia-reperfusion, and indicated that GK and GKRP may be necessary for neuronal survival against transient cerebral ischemia.

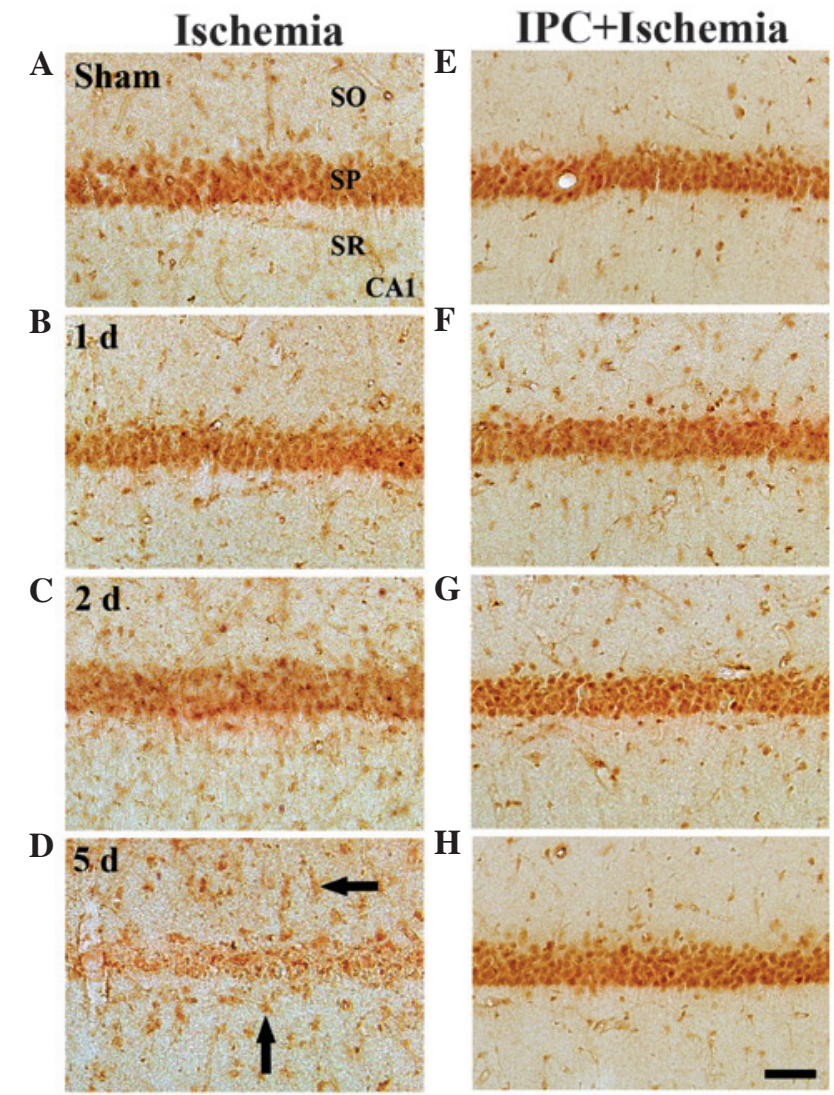

Figure 4. Immunohistochemistry for GKRP in the CA1 region of the (A-D) ischemia and (E-H) IPC + ischemia groups following sham surgery and 1, 2 and 5 days post-ischemia-reperfusion. GKRP immunoreactivity was readily detected in the SP in the sham-group. GKRP immunoreactivity in the SP was marginally decreased 2 days after ischemia-reperfusion. At 5 days post-ischemia, GKRP immunoreactivity was almost undetectable in the SP. At 5 days post- ischemia-reperfusion, marked GKRP immunoreactivity was observed in non-pyramidal cells (arrows) of the SO and SR. In the IPC + sham and IPC + ischemia groups, GKRP immunoreactivity was similar to that in the sham group. Scale Bar=50 $\mu \mathrm{m}$. GKRP, glucokinase regulatory protein; IPC, ischemic preconditioning; SP, stratum pyramidale; $\mathrm{SO}$; stratum oriens, SR; stratum radiatum.

The concept of IPC is defined as a brief, non-injurious episode of ischemia, which can protect the brain cells from a subsequent longer ischemic insult $(10,11)$. The first description of IPC in the brain was reported in a study by Kitagawa et al (30) in a gerbil model of global ischemia. Further studies have been performed in other animal models, including global and focal cerebral ischemia (12,31-35). The preconditioning time-period used in the present study was determined by that described in a previous report (27), which indicated that at least a 1 day interval between the sublethal $2 \mathrm{~min}$ ischemia and lethal $5 \mathrm{~min}$ ischemia is necessary for induction of the neuroprotection of the CA1 pyramidal cells. This protective mechanism has been termed 'ischemic tolerance,', however, the molecular mechanisms underlying ischemic tolerance remain to be fully elucidated. In the present study, a significantly protective effect of IPC against delayed neuronal death was observed in the hippocampal CA1 region following $5 \mathrm{~min}$ of transient cerebral ischemia in gerbils. At 5 days post-ischemic insult, pyramidal neurons in the $\mathrm{CA} 1$ region, but not the $\mathrm{CA} 2 / 3$, region exhibited features of neuronal death, and significant reductions in the numbers 
of $\mathrm{CV}^{+}$and $\mathrm{NeuN}^{+}$neurons and the appearance of F-J B neurons were observed.

In the present study, the endogenous expression levels of GK and GKRP in the CA1 region were compared between the ischemia group and the IPC + ischemia group and found that the immunoreactivities of GK and GKRP were well-detected in the pyramidal neurons of the CA1-3 regions, although the immunoreactivities were significantly decreased in the ischemic CA1 region following ischemia-reperfusion. Therefore, it is likely that GK and GKRP in the pyramidal neurons, which are the principal cells of the hippocampus, may be important in glucose metabolism. This finding is supported by a previous study that reported that the immunoreactivity of glucagon-like peptide-1 receptor (GLP-1R) is decreased in the gerbil hippocampal CA1 region 2 days after ischemia-reperfusion (36). It has also been observed that GLP-1R stimulation with exendin-4 leads to a protective effect against ischemic damage in the hippocampal CA1 region following transient cerebral ischemia (36). On the basis of the previous and present findings, it is likely that the ischemia-induced alteration of glucose metabolism may be associated with delayed neuronal death in the hippocampal CA1 region following transient cerebral ischemia. In addition, the maintained GK and GKRP immunoreactivities following IPC in the present study reflected potential survival mechanisms of the CA1 pyramidal neurons following transient ischemic injury.

Cerebral ischemia leads to the alteration of energy metabolism in the brain (37). It is well known that transient cerebral ischemia induces the modification of glucose metabolism in the hippocampal CA1 region, and it is associated with the development of neuronal cell damage and death (38). As neurons require a constant supply of glucose for normal function, glucose is a major source of energy metabolism for the central nervous system (39). Several studies have documented the effect of glucose-sensing neuropeptides on the development of post-ischemic glucose dysfunction and neuronal damage $(22,23)$, and evidence from a previous in vitro study revealed that glucose itself promotes the development of caspase-dependent apoptosis during re-oxygenation following oxygen and glucose deprivation, and promotes the production of reactive oxygen species, including superoxide anion, hydrogen peroxide and peroxynitrite (40). GK and GKRP have important regulatory roles in glucose metabolism as a glucose sensor. Alvarez et al (18) reported that the presence of GK interactions with GKRP in the rat hypothalamus may be involved in glucose sensing process and metabolic regulation. It has also been reported that GK modulates the activity of ATP-sensitive $\mathrm{K}+$ channels and determines the rate of cell firing (41). In addition, Mies et al (42) demonstrated that ATP levels in the CA1 region are unchanged between $6 \mathrm{~h}$ and 2 days post-ischemia, but reduce to $\sim 70 \% 4$ days post-ischemia. Therefore, GK can control glycolysis and may be a major candidate as a regulator of ATP production and $\mathrm{K}_{\text {ATP }}$ channel activity (41).

In conclusion, the present study demonstrated that IPC maintained the immunoreactivities of GK and GKRP in the CA1 pyramidal neurons following $5 \mathrm{~min}$ of transient ischemia, and that CA1 pyramidal neurons death did not occur following ischemia-reperfusion. These results suggested that GK and GKRP may be involved in protecting neurons from ischemia-reperfusion injury and that maintained GK and GKRP expression by IPC in the ischemic CA1 region may be a fundamental mechanism for survival of neurons after transient cerebral ischemia.

\section{Acknowledgements}

The authors would like to thank Mr. Seung Uk Lee for their technical assistance. This work was supported by the Basic Science Research Program through the National Research Foundation (NRF) of Korea, funded by the Ministry of Science, ICT and future Planning (grant. no. NRF-2014R1A2A2A0100 5307) and by the NRF of Korea, funded by the Ministry of Education, Science and Technology (grant. no. 2010-0010580).

\section{References}

1. Neigh GN, Glasper ER, Kofler J, Traystman RJ, Mervis RF, Bachstetter A and DeVries AC: Cardiac arrest with cardiopulmonary resuscitation reduces dendritic spine density in CA1 pyramidal cells and selectively alters acquisition of spatial memory. Eur J Neurosci 20: 1865-1872, 2004.

2. Schneider A, Böttiger BW and Popp E: Cerebral resuscitation after cardiocirculatory arrest. Anesth Analg 108: 971-979, 2009.

3. Harukuni I and Bhardwaj A: Mechanisms of brain injury after global cerebral ischemia. Neurol Clin 24: 1-21, 2006.

4. Kirino T and Sano K: Selective vulnerability in the gerbil hippocampus following transient ischemia. Acta Neuropathol 62: 201-208, 1984

5. Kirino T: Delayed neuronal death in the gerbil hippocampus following ischemia. Brain Res 239: 57-69, 1982.

6. Chan PH: Reactive oxygen radicals in signaling and damage in the ischemic brain. J Cereb Blood Flow Metab 21: 2-14, 2001

7. Won MH, Kang TC, Jeon GS, Lee JC, Kim DY, Choi EM, Lee KH, Choi CD, Chung MH and Cho SS: Immunohistochemical detection of oxidative DNA damage induced by ischemia-reperfusion insults in gerbil hippocampus in vivo. Brain Res 836: 70-78, 1999.

8. Won MH, Kang T, Park S, Jeon G, Kim Y, Seo JH, Choi E, Chung $\mathrm{M}$ and Cho SS: The alterations of N-Methyl-D-aspartate receptor expressions and oxidative DNA damage in the CA1 area at the early time after ischemia-reperfusion insult. Neurosci Lett 301: 139-142, 2001.

9. Lee CH, Park JH, Yoo KY, Choi JH, Hwang IK, Ryu PD, Kim DH, Kwon YG, Kim YM and Won MH: Pre- and post-treatments with escitalopram protect against experimental ischemic neuronal damage via regulation of BDNF expression and oxidative stress. Exp Neurol 229: 450-459, 2011.

10. Schmued LC and Hopkins KJ: Fluoro-Jade B: A high affinity fluorescent marker for the localization of neuronal degeneration. Brain Res 874: 123-130, 2000.

11. Lehotský J, Burda J, Danielisová V, Gottlieb M, Kaplan P and Saniová B: Ischemic tolerance: The mechanisms of neuroprotective strategy. Anatomical Rec (Hoboken) 292: 2002-2012, 2009.

12. Gidday JM: Cerebral preconditioning and ischaemic tolerance. Nat Rev Neurosci 7: 437-448, 2006.

13. Kardesoglu E, Isilak Z, Uz O and Yiginer O: Ischemic conditioning: A current concept in reducing reperfusion injury. Chin Med J (Engl) 124: 480, 2011.

14. Liang Y, Jetton TL, Zimmerman EC, Najafi H, Matschinsky FM and Magnuson MA: Effects of alternate RNA splicing on glucokinase isoform activities in the pancreatic islet, liver and pituitary. J Biol Chem 266: 6999-7007, 1991.

15. Maekawa F, Toyoda Y, Torii N, Miwa I, Thompson RC, Foster DL, Tsukahara S, Tsukamura $\mathrm{H}$ and Maeda K: Localization of glucokinase-like immunoreactivity in the rat lower brain stem: For possible location of brain glucose-sensing mechanisms. Endocrinology 141: 375-384, 2000.

16. Roncero I, Sanz C, Alvarez E, Vázquez P, Barrio PA and Blázquez E: Glucokinase and glucokinase regulatory proteins are functionally coexpressed before birth in the rat brain. $\mathrm{J}$ Neuroendocrinol 21: 973-981, 2009.

17. Roncero I, Alvarez E, Vázquez $\mathrm{P}$ and Blázquez E: Functional glucokinase isoforms are expressed in rat brain. J Neurochem 74: 1848-1857, 2000 . 
18. Alvarez E, Roncero I, Chowen JA, Vázquez P and Blázquez E: Evidence that glucokinase regulatory protein is expressed and interacts with glucokinase in rat brain. J Neurochem 80: 45-53, 2002.

19. Malaisse WJ, Malaisse-Lagae F, Davies DR, Vandercammen A and Van Schaftingen E: Regulation of glucokinase by a fructose-1-phosphate-sensitive protein in pancreatic islets. Eur J Biochem 190: 539-545, 1990

20. Van Schaftingen E: Short-term regulation of glucokinase. Diabetologia 37 (Suppl 2): S43-S47, 1994

21. Vandercammen A and Van Schaftingen E: The mechanism by which rat liver glucokinase is inhibited by the regulatory protein Eur J Biochem 191: 483-489, 1990.

22. Harada S, Fujita-Hamabe W and Tokuyama S: Ischemic stroke and glucose intolerance: A review of the evidence and exploration of novel therapeutic targets. J Pharmacol Sci 118: 1-13, 2012.

23. Wang YY, Lin SY, Chuang YH, Chen CJ, Tung KC and Sheu WH: Adipose proinflammatory cytokine expression through sympathetic system is associated with hyperglycemia and insulin resistance in a rat ischemic stroke model. Am J Physiol Endocrinol Metab 300: E155-E163, 2011.

24. Park JH, Lee CH, Kim IH, Ahn JH, Cho JH, Yan BC, Lee JC, Lee TH, Seo JY, Cho JH, et al: Time-course changes in immunoreactivities of glucokinase and glucokinase regulatory protein in the gerbil hippocampus following transient cerebral ischemia. Neurochem Res 38: 2640-2649, 2013.

25. Liu YR, Lei RY, Wang CE, Zhang BA, Lu H, Zhu HC and Zhang GB: Effects of catalpol on ATPase and amino acids in gerbils with cerebral ischemia/reperfusion injury. Neurol Sci 35: 1229-1233, 2014.

26. Wang L, Zhu QL, Wang GZ, Deng TZ, Chen R, Liu MH and Wang SW: The protective roles of mitochondrial ATP-sensitive potassium channels during hypoxia-ischemia-reperfusion in brain. Neurosci Lett 491: 63-67, 2011.

27. Nakamura H, Katsumata T, Nishiyama Y, Otori T, Katsura K and Katayama Y: Effect of ischemic preconditioning on cerebral blood flow after subsequent lethal ischemia in gerbils. Life Sci 78: 1713-1719, 2006.

28. Candelario-Jalil E, Alvarez D, Merino N and León OS: Delayed treatment with nimesulide reduces measures of oxidative stress following global ischemic brain injury in gerbils. Neurosci Res 47: 245-253, 2003.

29. Lee CH, Park JH, Choi JH, Yoo KY, Ryu PD and Won MH: Heat shock protein 90 and its cochaperone, p23, are markedly increased in the aged gerbil hippocampus. Exp Gerontol 46 768-772, 2011.
30. Kitagawa K, Matsumoto M, Kuwabara K, Tagaya M, Ohtsuki T, Hata R, Ueda H, Handa N, Kimura K and Kamada T: 'Ischemic tolerance' phenomenon detected in various brain regions. Brain Res 561: 203-211, 1991

31. Liu Y, Kato H, Nakata N and Kogure K: Protection of rat hippocampus against ischemic neuronal damage by pretreatment with sublethal ischemia. Brain Res 586: 121-124, 1992.

32. Kirino T, Tsujita Y and Tamura A: Induced tolerance to ischemia in gerbil hippocampal neurons. J Cereb Blood Flow Metab 11: 299-307, 1991.

33. Nishi S, Taki W, Uemura Y,Higashi T, Kikuchi H, Kudoh H, Satoh M and Nagata K: Ischemic tolerance due to the induction of HSP70 in a rat ischemic recirculation model. Brain Res 615: 281-288, 1993.

34. Toyoda T, Kassell NF and Lee KS: Induction of ischemic tolerance and antioxidant activity by brief focal ischemia. Neuroreport 8: 847-851, 1997.

35. Stagliano NE, Pérez-Pinzón MA, Moskowitz MA and Huang PL: Focal ischemic preconditioning induces rapid tolerance to middle cerebral artery occlusion in mice. J Cereb Blood Flow Metab 19: 757-761, 1999.

36. Lee CH, Yan B, Yoo KY, Choi JH, Kwon SH, Her S, Sohn Y, Hwang IK, Cho JH, Kim YM, et al: Ischemia-induced changes in glucagon-like peptide-1 receptor and neuroprotective effect of its agonist, exendin-4, in experimental transient cerebral ischemia. J Neurosci Res 89: 1103-1113, 2011.

37. Dirnagl U, Iadecola C and Moskowitz MA: Pathobiology of ischaemic stroke: An integrated view. Trends Neurosci 22: 391-397, 1999.

38. Jorgensen MB, Wright DC and Diemer NH: Postischemic glucose metabolism is modified in the hippocampal CA1 region depleted of excitatory input or pyramidal cells. J Cereb Blood Flow Metab 10: 243-251, 1990.

39. Levin BE: Glucosensing neurons do more than just sense glucose. Int J Obes Relat Metab Disord 25 (Suppl 5): S68-S72, 2001

40. Serra-Perez A, Verdaguer E, Planas AM and Santalucia T: Glucose promotes caspase-dependent delayed cell death after a transient episode of oxygen and glucose deprivation in SH-SY5Y cells. J Neurochem 106: 1237-1247, 2008.

41. Dunn-Meynell AA, Routh VH, Kang L, Gaspers L and Levin BE: Glucokinase is the likely mediator of glucosensing in both glucose-excited and glucose-inhibited central neurons. Diabetes 51: 2056-2065, 2002.

42. Mies G, Paschen W and Hossmann KA: Cerebral blood flow, glucose utilization, regional glucose and ATP content during the maturation period of delayed ischemic injury in gerbil brain. J Cereb Blood Flow Metab 10: 638-645, 1990. 